\title{
ESTRATEGIA PEDAGÓGICA PARA LA ORIENTACIÓN PROFESIONAL EN LA EDUCACIÓN PREUNIVERSITARIA
}

PhD. Romelio Basto Castillo

Universidad de Oriente

rbasto@uo.edu.cu
PhD. Miguel Basto Rizo Universidad de Oriente mabasto@uo.edu.cu
Palabras claves: orientación profesional, formación continua, trabajo metodológico.

Keywords: professional orientation, continuous formation, methodological work.
Recibido: 11 de enero de 2017

Aceptado: 08 de febrero de 2017

\section{RESUMEN}

El desarrollo de la orientación profesional con adolescentes y jóvenes en el preuniversitario, contribuye al cumplimiento del proyecto educativo de centro, por lo que en la actualidad exige de los directivos, docentes, la escuela, la familia y la comunidad una atención priorizada y una reconceptualización en el orden científico, técnico, metodológico e ideopolítico, que influya en la elección profesional hacia las carreras necesarias para el desarrollo económico-social, y especialmente del territorio, como condición estratégica para el desarrollo del país, de ahí la importancia social de la orientación profesional. Resultado de estudios de Doctorado, el autor del presente artículo exhibe una estrategia pedagógica para la orientación profesional en la Educación Preuniversitaria. El uso de métodos del nivel teórico y empírico sirvió de base en su construcción y constatación de su factibilidad.

\section{ABSTRACT}

The development of the professional orientation with adolescents and young in Pre-University Education, it contributes to the execution of the educational project of center, for that that at the present time demands of the directive, educational, the school, the family and the community a prioritized attention and a reconceptualization in the scientific order, technician, methodological and ide politico that influences in the professional election toward the necessary careers for the socio-economic development, and especially of the territory, as strategic condition for the development of the country, of there the social importance of the professional orientation. Result of studies of Doctorate, the author of the present article exhibits a pedagogic strategy for the professional orientation in the PreUniversity Education. The use of methods of the theoretical and empiric level served as base in its construction and verification of its feasibility. 


\section{INTRODUCCIÓN}

En la actualidad, la situación que presenta la elección de las profesiones por el estudiante al culminar el preuniversitario, refuerza la misión del docente sea más intensa en lo ideopolítico, y psicopedagógico; tanto es así que en el contexto del proceso de preparación profesional pedagógica recibe para el perfeccionamiento de su desempeño profesional un sistema de influencias, que deben contribuir a que pueda desarrollar un intenso y eficiente proceso de educación de la personalidad.

Consideración asumida al resaltar la función orientadora del docente sobre el grupo y los estudiantes, lo que debe revertirse también en la orientación profesional hacia y en la familia, la comunidad y las organizaciones político estudiantiles, de manera que por la importancia de estos factores, haya una incidencia educativa integrada y coherente.

En tal sentido, y como parte de los elementos necesarios e indispensables que debe tener en cuenta el docente para realizar una adecuada orientación profesional, desde la clase, como forma fundamental del proceso de enseñanza- aprendizaje, se debe considerar lo que González (1989) establece como una nueva formación psicológica compleja a la que le da el nombre de Intenciones Profesionales que no es más que la constatación en algunos estudiantes de un nivel superior de desarrollo de la motivación profesional. Las Intenciones Profesionales presuponen "la elaboración personal del proyecto profesional asumido, lo que integran los conocimientos del adolescente sobre la profesión y las principales emociones vinculadas con las necesidades y motivos que se expresan en la tendencia orientadora hacia la profesión" (citado por Álvarez,2001, p. 23).

Es pertinente considerar que una sólida preparación profesional pedagógica de los docentes, en orientación profesional, contribuye al cumplimiento de la misión social del preuniversitario, como manifestación de los docentes, de un desempeño profesional efectivo, ya que se logra mayor conciencia en el educando para tomar la decisión de elegir su futura profesión, en el final de la adolescencia y principio de la juventud, por lo que se coincide con González Rey F. cuando plantea que: "(...) una adecuada educación de la personalidad en su aspecto profesional contribuirá a que en el acto de autodeterminación para adolescentes y jóvenes (...) se sientan verdaderamente comprometidos e implicados como personalidad en su decisión" (González ,1993, págs. 3 y 4).

Por eso, se entiende que la orientación profesional está concebida como un proceso sistemático y sistémico, que tiene de forma general su expresión en el proceso de enseñanza- aprendizaje y, específicamente, en la clase con el fin de establecer un vínculo adecuado de la educación con la vida, de manera que contribuya a su preparar al hombre para la vida, como reza el principio martiano muy significativo y que fundamenta la pedagogía socialista cubana, y a lo cual, el proceso de preparación profesional pedagógica de los docentes, en orientación profesional, brinda respuestas.

Se propone una estrategia pedagógica para la orientación profesional en la educación preuniversitaria, resultado de profundización de estudios doctoral en la enseñanza antes mencionada. 


\section{DESARROLLO}

Para la elaboración de la estrategia pedagógica propuesta se han tenido en cuenta diferentes definiciones o criterios expresados por varios autores. Para los fines del presente artículo la estrategia pedagógica se asume como: "La concepción teóricopráctica de la dirección del proceso pedagógico durante la transformación del estado real al estado deseado, en la formación y desarrollo de la personalidad, de los sujetos de la educación, determinadas a partir del diagnóstico integral de las potencialidades y necesidades de los mismos que condiciona el sistema de acciones para alcanzar los objetivos, tanto en lo personal, lo grupal como en la institución escolar". (Sierra, 2004)

Esta estrategia pedagógica posee las siguientes características:

Carácter indagativo de las necesidades de preparación profesional pedagógica de los docentes, en orientación profesional.

$\checkmark$ Carácter flexible, susceptible a las modificaciones y adecuaciones en correspondencia con las características de la escuela, el colectivo de profesores y el entorno en que se desarrolla.

$\checkmark$ Carácter proyectivo, sistematizador y desarrollador de la proyección de la orientación profesional según las dificultades o problemas detectados a través del diagnóstico.

$\checkmark$ Carácter planificado a través de lo instructivo, lo educativo y lo desarrollador, donde el docente estimule la participación del resto de las influencias educativas.

$\checkmark$ La contrastabilidad a partir de la valoración y la evaluación para determinar la aproximación al cumplimiento de los objetivos trazados en cada una de las acciones.

$\checkmark$ Posibilita la participación y el protagonismo de todos los factores en las diferentes acciones y en la toma de decisiones dentro el marco de la estrategia para integrar el querer, el saber y el poder.

$\checkmark$ Posee un balance coherente de las acciones consideradas como totalidad.

Se significa, que es importante tener en cuenta, que en la estrategia pedagógica su comprensión e interpretación por todos los factores dependerá del papel de las acciones, pasos y consecuencias para obtener resultados satisfactorios y tiene como objetivo: elevar la calidad de la formación continua de los docentes con un carácter sistémico y desarrollador que se manifieste en el crecimiento profesional pedagógico como un nivel superior de la preparación profesional pedagógica de los docentes, en orientación profesional en el preuniversitario.

Posee un basamento en las siguientes premisas:

$\checkmark$ El vínculo estrecho entre la escuela preuniversitaria, sus docentes, sus directivos, la familia y los factores de la comunidad.

$\checkmark$ Concebir a la preparación profesional pedagógica del docente, en orientación profesional como condición esencial que contribuya a dinamizar el encargo social de la Educación Preuniversitaria dando respuesta a los objetivos de la educación integral y preprofesional. 
Considerar a la preparación profesional pedagógica de los docentes, en la orientación profesional como un proceso que provoca un crecimiento profesional y se enmarca dentro de las exigencias de toda actividad humana y particularmente como actividad pedagógica desarrolladora.

Exigencias metodológicas en las que se sustenta el proceso de preparación profesional pedagógica de los docentes, en orientación profesional:

1) Expresión de la integralidad de lo instructivo, educativo y desarrollador presentes en la preparación profesional pedagógica de los docentes, en orientación profesional con manifestación en las relaciones establecidas entre las configuraciones, dimensiones y eslabones del proceso de preparación.

2) Que los docentes, al crecer profesionalmente, puedan poner en práctica el proceso de enseñanza- aprendizaje, para dar salida efectiva y eficaz a la orientación profesional desde la clase, como forma fundamental para la búsqueda de respuestas efectiva a las exigencias sociales en la formación de los estudiantes preuniversitarios, por el carácter estratégico que ello tiene para el desarrollo económico del país.

3) Incorporar una visión desarrolladora del proceso de preparación profesional pedagógica de los docentes, basado en la integración del trabajo metodológico, la superación profesional y la investigación pedagógica con la aplicación de la ciencia, la tecnología e innovación pedagógica.

4) Responde a una planificación que deja establecido objetivos a corto y mediano plazo para transformar al objeto desde su estado real hasta el estado deseado a partir de las etapas, acciones, recursos, medios y métodos que corresponden a estos objetivos.

\section{Etapas y acciones de la estrategia pedagógica}

La estrategia pedagógica para el proceso de preparación profesional pedagógica de los docentes, en orientación profesional, desde la labor del departamento docente en el preuniversitario cuenta con tres etapas, éstas son:

1- Etapa de diagnóstico de los docentes para la preparación profesional pedagógica en orientación profesional desde la labor del departamento docente

2- Etapa de ejecución del proceso de preparación profesional pedagógica de los docentes, en orientación profesional

3- Etapa de evaluación

Cada una de ellas con sus respectivas acciones describiéndose de la siguiente forma:

1-Etapa de diagnóstico de los docentes para la preparación profesional pedagógica en orientación profesional, desde la labor del departamento docente

Esta etapa a través de un diagnóstico, se proyecta hacia los aspectos que evidencian la necesidad de preparar profesionalmente a los docentes para llevar a cabo la orientación profesional desde el punto de vista metodológico, donde la identificación contextual de necesidades es expresión del resultado de dicho diagnóstico de necesidades de preparación profesional pedagógica de los docentes en el contexto de su actuación profesional. 
Esta es la base de partida del diseño y planificación de las acciones para favorecer la preparación profesional pedagógica de los docentes del preuniversitario desde la integración del trabajo metodológico, la superación profesional y la investigación pedagógica. Es inherente al desarrollo de esta primera etapa, la caracterización del claustro en cada departamento docente, la jerarquización de los problemas y necesidades de preparación profesional pedagógica en orientación profesional, se utiliza la información que aportan los diversos instrumentos aplicados, para favorecer la posibilidad de transformar la realidad que se diagnostica.

Acción 1.1: Diagnóstico de las necesidades de preparación profesional pedagógica de los docentes, en orientación profesional.

Se realiza el diagnóstico donde se determinan las potencialidades y necesidades de superación profesional de los docentes de cada uno de los departamentos docentes del preuniversitario, desde el punto de vista teórico y práctico, para la preparación profesional pedagógica en orientación profesional con el propósito de contextualizar la preparación que demanda este personal y analizar los documentos necesarios a tal efecto.

Se debe considerar como premisa, que el diagnóstico constituye un proceso comunicativo, continuo y coherente, que posibilita comprender y explicar el nivel de preparación de los docentes y las características del contexto escolar, así como asumir determinada decisión en relación con la transformación que en ellos se desea lograr, en este caso, sobre la orientación profesional. Se debe tener en cuenta, que el diagnóstico se sustenta en las interacciones, diálogos e intercambios entre los sujetos que en él participan, así como en la necesidad de un clima favorable, para propiciar que sea mayor y más certera la información ofrecida.

Acción 1.2: Caracterización de los docentes que recibirán la influencia del proceso de preparación profesional pedagógica, en orientación profesional.

En esta acción se caracteriza el claustro de profesores, de los departamentos docentes del preuniversitario, en cuanto a su composición por categorías docentes y científicas, años de experiencia, formación profesional, experiencia profesional en la educación en cuestión y en cada grado, resultados de las evaluaciones docentes y de las investigaciones realizadas, tiempo y disponibilidad de los docentes para llevar a cabo la superación. Se sustenta en la acción anterior, al tomar en consideración la información que aporta el diagnóstico, de manera que considera necesariamente los conocimientos, las motivaciones, los intereses y las necesidades de superación profesional de los docentes.

Acción 1.3: Identificación de los problemas y necesidades de preparación profesional pedagógica de los docentes, en orientación profesional.

Se realiza un análisis con el claustro de docentes donde se evalúan los resultados del diagnóstico de identificación de necesidades aplicado dentro del marco de la estrategia y se ubican por orden de prioridad los problemas y las necesidades de superación profesional, a partir del propio desarrollo de la actividad práctica que realizan en la dinámica del proceso de enseñanza- aprendizaje en el preuniversitario.

Acción 1.4: Diseño de las actividades del proceso de preparación profesional pedagógica de los docentes, en orientación profesional. 
En el desarrollo de esta acción se debe garantizar la planificación organizada y eficaz por parte del departamento docente y la institución responsabilizada con la superación profesional, en la Universidad de Oriente, que se ocupa de las actividades que luego serán ejecutadas como parte de la implementación de la Estrategia pedagógica que se expone, a partir de la determinación de los objetivos, contenidos, métodos, medios a emplear, actores y formas organizativas en cada momento, además de la evaluación sistemática y final, desarrolladas para constatar los cambios que se manifiestan en los docentes como consecuencias del trabajo metodológico, la superación profesional e investigación educacional desarrollados.

El diseño o concepción de las actividades previstas a corto plazo, se concretan en:

$\checkmark$ Conferencias

$\checkmark$ Talleres

$\checkmark$ Seminario

Estas acciones le imprimen una identidad propia al proceso de preparación profesional pedagógica de los docentes, contribuyendo al intercambio, la reflexión y el desarrollo eficaz del trabajo metodológico, la superación profesional e investigación pedagógica en un marco colectivo e individual.

2-Etapa de ejecución del proceso de preparación profesional pedagógica de los docentes, en orientación profesional

En esta etapa, se desarrolla el proceso de preparación profesional pedagógica de los docentes, en orientación profesional. El objetivo es instrumentar en la práctica las acciones diseñadas en la primera etapa, con vista al perfeccionamiento del proceso de preparación profesional pedagógica de los docentes, a partir de la integración de la superación profesional con el trabajo metodológico y la investigación pedagógica, como expresión de la labor del departamento docente.

Al profundizar en la esencia de la configuración proyección personalizada de necesidades, esta se identifica con relación dialéctica a determinados contenidos teóricos y prácticos que son necesarios tener en cuenta para el desarrollo del proceso de preparación profesional pedagógica de los docentes a fin de enfrentar la orientación profesional. Dichos contenidos establecen la sistematización metodológica e investigativa-pedagógica, en el proceso con un carácter específico en la orientación profesional con relación dialéctica, como esencia de la integración del trabajo metodológico, la superación profesional y la investigación pedagógica como expresión identitaria.

Al implicar a los docentes en el sistema de acciones que se derivan de las actividades de superación profesional planteadas, se propicia la adquisición de forma consciente de los elementos necesarios y suficientes para desarrollar la orientación profesional dirigida por el departamento docente. De esta forma, se logra una participación activa y reflexiva por parte de los docentes, con un papel protagónico.

Acción 2.1: Presentación de las diferentes formas organizativas del proceso de preparación profesional pedagógica de los docentes, en orientación profesional.

En esta acción se les presenta a los docentes las actividades de preparación profesional pedagógica a corto y mediano plazo, con carácter específico en orientación profesional. Este momento es muy importante desde el punto de vista 
intelectual, por tanto, los docentes deben dar muestra de su creatividad y dominio del contenido de manera, que se logre establecer la relación entre las asignaturas y el contenido propio de la orientación profesional.

Teniendo en cuenta la etapa anterior, a partir de las motivaciones, intereses e inquietudes originadas, el docente identifica el problema, reflexiona y asume una posición desde su experiencia individual; busca alternativas en cuanto a cuál debe ser la respuesta, solución o posición a tener en cuenta, al considerar las razones que fundamentan su visión individual y del colectivo.

El pensamiento del docente se orienta hacia la búsqueda de las causas y efectos para lo cual debe realizar algunas operaciones lógicas como: la comparación, el análisis, la síntesis, entre otras. Es un proceso de hallazgos de argumentos frente a la situación planteada, con el objetivo de resolverla, sobre la base de conocimientos y razonamientos determinados, o nuevos modos de acción.

Acción 2.2: Argumentación de las diferentes formas organizativas del proceso de preparación profesional pedagógica de los docentes, en orientación profesional.

En este momento, debe aportarse al docente todos los elementos necesarios con los que debe operar para la correcta preparación profesional pedagógica, en orientación profesional. Para ello, en la argumentación de la situación problémica deben aclararse conceptos que se introducen, y definirse los aspectos más significativos o relevantes mediante una incitación a la búsqueda. En este sentido, esas situaciones problémicas y los resultados científicos investigativos alcanzados constituyen una herramienta fundamental para estimular la búsqueda y el descubrimiento de los problemas existentes, y que frenan el desarrollo de la orientación profesional, lo cual se realiza mediante un proceso de reflexión.

\section{Acción 2.3: Dirección del debate.}

En esta acción se debe propiciar el intercambio entre los docentes. Se debe crear un clima favorable, considerando que todas las respuestas sean válidas, mediante la ejercitación de la capacidad de escucha, cooperación, tolerancia, solidaridad y diálogo en la situación planteada, y deben considerarse las posibles respuestas ante las diferentes posiciones, con antelación.

Se debe tomar en consideración, que lo importante es buscar las razones que hay detrás de cada respuesta para eliminar sus dudas e impulsarlos a tomar decisiones. Interrogar, cuestionar constituyen elementos de reflexión que viabilizan todas las vías y formas de expresión de los docentes de manera espontánea, pues debe considerar que el diálogo interior con él mismo ya favorece su desarrollo.

En función de esta acción, en el transcurso de la actividad, se debe retroalimentar al docente con el reconocimiento positivo de sus aportaciones y acciones, al reconocer sus errores y limitaciones en el proceso de preparación profesional pedagógica, en orientación profesional, lo que potencia su identidad y autoestima.

El docente debe precisar qué se ha logrado con el desarrollo de la actividad, lo cual le permite avanzar en sus criterios y reconsiderar su actuación para continuar ascendiendo a un estadio superior. Finalmente, se puede llegar a un consenso o a una respuesta única. En este caso, lo que se debe considerar es la importancia del debate y la práctica de escuchar argumentos y razones del otro participante, con atención en el proceso de preparación, durante un clima de respeto y ayuda mutua. 


\section{3-Etapa de evaluación}

Esta etapa da cuenta de la funcionalidad de la estrategia, de la pertinencia de las acciones y revela la calidad de la preparación profesional pedagógica de los docentes, para desarrollar la orientación profesional, desde la labor del departamento docente, en el preuniversitario. El objetivo es evaluar el dominio de los conocimientos y habilidades necesarios alcanzados por las docentes para la orientación profesional, a partir de la implementación de las acciones de preparación profesional pedagógica concebidas.

Se brinda una evaluación de la transformación alcanzada por los docentes de cada departamento docente, a partir de perfeccionar su desempeño profesional, tomando como referencia los momentos de cambios propuestos a tal efecto, como expresión del crecimiento profesional pedagógico de los docentes, en orientación profesional. El crecimiento profesional pedagógico fortalece lo expresado en el modelo del profesional de la educación para el preuniversitario, donde aparecen las tres responsabilidades que tiene el profesor en la escuela: la docente-metodológica, la orientadora educativa y la investigativa y de superación, ya que la escuela preuniversitaria es decisiva en la formación de los futuros trabajadores, técnicos y profesionales para enfrentar los retos del desarrollo en el país con una visión más integradora del mundo.

En el proceso de evaluación de la Estrategia pedagógica se conciben tres formas fundamentales: la autoevaluación; la heteroevaluación y la coevaluación.

La autoevaluación se resume en los criterios autovalorativos por los docentes del departamento docente implicados en el proceso, ya que, al tenerlos como protagonistas, contribuyen a la toma de conciencia y al compromiso con la sociedad, a través de la valoración que cada cual realiza de su propia participación en el desarrollo del proceso. Se estructura como una evaluación polivalente al recopilar información a lo largo del proceso de implementación de la estrategia pedagógica para la preparación profesional pedagógica, mediante diferentes técnicas (auto observación y guía para la autoevaluación). El docente se autoevalúa con el propósito de determinar su grado de aportación al proceso.

La heteroevaluación es la evaluación exterior, expresada en la estimación que realizan otros sujetos que no estén implicados en la Estrategia pedagógica, al tratar de valorar en qué medida se favorece el proceso de preparación profesional pedagógica, cuyo objetivo esencial es lograr el crecimiento profesional pedagógico en este aspecto, para la formación integral y preprofesional de los estudiantes de este nivel educacional. Entre los mecanismos que se proponen está la entrevista a los docentes para conocer su nivel de satisfacción en el desarrollo del proceso; la observación a las actividades que se realizan, como principal mecanismo de la heteroevaluación.

La coevaluación es el procedimiento que se refiere a procesos valorativos de carácter cruzado. Es una evaluación cooperativa y solidaria, centrada en lo positivo como vía para mejorar las limitaciones e insuficiencias en la realización de las acciones de preparación profesional pedagógica de los docentes, en orientación profesional, que exigen de la participación los docentes implicados, los cuales son objeto y sujetos de valoración. 
Con una visión integradora, se ofrece una evaluación del estado de transformación alcanzado por los participantes en el proceso de preparación profesional pedagógica, como expresión de su crecimiento profesional pedagógico, el que se constituye en la vía principal para mejorar su desempeño profesional, tomando como referencia los momentos de cambios propuestos a tal efecto. La evaluación continua y sistemática del desarrollo que estos van alcanzando, les posibilita autoevaluar, evaluar y coevaluar los momentos de cambios propuestos, teniendo en cuenta los procesos metacognitivos, donde demuestran conocimientos de sus avances, estancamientos o retrocesos, que, a tal efecto, puede convertirse en una motivación para alcanzar metas superiores y lograr un desarrollo profesional superior.

La evaluación de la estrategia pedagógica se realiza en el proceso de implementación y se efectúa en cada uno de los niveles y las acciones de preparación profesional pedagógica de manera integrada.

Con la utilización del método estudio de caso, se clarifican los aspectos que corroboran la pertinencia de la estrategia pedagógica, lo que se enmarca en el tipo de investigación asumida de carácter cualitativa, como una contribución de gran significado para provocar el crecimiento profesional pedagógico de los docentes y así perfeccionar la orientación profesional desde la labor del departamento docente en el preuniversitario.

El estudio de caso propuesto se ha configurado en torno a diferentes aspectos asociados a la orientación profesional; todos ellos íntimamente relacionados entre sí, considerando que su aplicación servirá para reflexionar sobre su introducción en la práctica escolar a fin de lograr mejoras en el proceso objeto de estudio, por lo que la atención, de manera particular, se constata de manera integral y holística, la utilidad de los aportes de la investigación realizada, particularmente la estrategia, a partir de una concepción humanista de la educación.

En correspondencia con criterios establecidos por diversos autores, el estudio de caso se puede estructurar sobre la base de tres fases, identificadas como: preactiva, interactiva y postactiva.

Tipificación del estudio de caso:

El estudio de caso asumido es del tipo evaluativo según Guba y Lincoln (1981) citado por (Cebreiro y Fernández, 2004), lo que implica descripción, explicación y juicio a partir de las diversas informaciones con que se puede contar, lo que posibilitará una descripción amplia, diversa y profunda del problema que se aborda, lo cual le dará un carácter holístico ya que la información de la cual se dispone es la que posibilita emitir un juicio evaluativo con la calidad adecuada, en tanto la emisión de juicios es el acto final y esencial de la evaluación realizada dentro del marco del estudio realizado.

La factibilidad se despliega por la obtención de resultados cada vez más acertados en relación con los objetivos generales y específicos de la estrategia pedagógica que se manifiesta a partir de la relación de los análisis de los indicadores y acciones estratégicas elaboradas para el proceso de preparación profesional pedagógica de los docentes, en orientación profesional, desde la integración del trabajo metodológico, la superación profesional y la investigación pedagógica. 
Las acciones de la estrategia pedagógica se dinamizaron a través del desarrollo de diferentes formas organizativas: conferencia introductoria, talleres de preparación, un seminario y un diplomado. Se cumplieron en todos los casos las exigencias de lo establecido en las reglamentaciones para el desarrollo del trabajo metodológico y la superación profesional.

En tal sentido, las principales observaciones de los directivos y docentes que conforman el grupo de profesionales seleccionados, dieron cuenta de la eficacia de la estrategia, con manifestación en:

Los jefes del primer nivel y jefes de departamentos mostraron: preparación teóricometodológica y capacidad para dirigir el proceso de preparación profesional pedagógica de los docentes, en orientación profesional, desde la integración del trabajo metodológico, la superación profesional y la investigación pedagógica e identificación, con relación a la necesidad de consolidar la integración entre el preuniversitario y la Universidad de Oriente, para el desarrollo del proceso desde la integración ya prevista.

Los docentes principales de asignaturas mostraron: preparación teóricometodológica para impulsar en su radio de acción el proceso de preparación profesional pedagógica, en orientación profesional y la comprensión de la necesidad de su participación activa en dicho proceso desde la integración del trabajo metodológico, la superación profesional y la investigación pedagógica como exigencia profesional de la labor que desempeñan.

Además, se evidente la comprensión de la salida profesional de lo asimilado como consecuencia de la influencia de este proceso, que tiene que ser a través de la clase, donde el trabajo metodológico tiene un papel relevante para lograr el adecuado vínculo del contenido de cada asignatura con la orientación profesional e identificación con relación a la necesidad de consolidar la integración entre el preuniversitario y la Facultad de Educación en Ciencias Sociales y Humanidades de la Universidad de Oriente para el desarrollo del proceso de preparación profesional pedagógica de los docentes, en orientación profesional.

La funcionalidad se constató a partir del nivel de comprensión y convencimiento de la validez de la coherencia existente en los eslabones, dimensiones y configuraciones que describen el proceso de preparación profesional pedagógica de los docentes, en orientación profesional; carácter transformador se puso de manifiesto en el papel asumido por los docentes que recibieron la influencia de la Estrategia pedagógica, dado el crecimiento profesional pedagógico desarrollado, lo que posibilitará enfrentar la orientación profesional con un desempeño profesional superior, perfeccionándose así este trabajo de tan significativa importancia en el preuniversitario.

Finalmente, se expresa que el cumplimiento de estas fases permitió perfeccionar la estrategia pedagógica propuesta para el desarrollo de la orientación profesional en la educación preuniversitaria, con una expresión instructiva, formativa y desarrolladora. 


\section{CONCLUSIONES}

Los aspectos antes abordados permiten arribar a las siguientes conclusiones:

$\checkmark$ Con la elaboración e instrumentación de la estrategia pedagógica se logra perfeccionar la preparación profesional pedagógica de los docentes, resultado de la interpretación realizada a partir de su estructura, para el cumplimiento del objetivo la cual se dinamiza a partir del diagnóstico realizado que permite descubrir las necesidades específicas en el objeto de estudio abordado.

$\checkmark$ En el estudio de caso desarrollado, se valoró de satisfactoria la calidad, factibilidad, validez y coherencia de la estrategia, que constituye un factor que puede contribuir al perfeccionamiento de la orientación profesional, y así alcanzar la efectividad de este trabajo para lograr el cumplimiento del fin de la Educación Preuniversitaria. 


\section{REFERENCIAS BIBLIOGRÁFICAS}

Álvarez, A. (2001). El trabajo de formación vocacional profesional: un reto pedagógico para el departamento docente en la enseñanza preuniversitaria. Tesis doctoral. Santiago de Cuba.

Basto, R. (2013). La preparación pedagógica de los profesores como factor de calidad educativa asociada al trabajo de orientación profesional desde la labor del departamento docente en la Educación Preuniversitaria. México: Colegio Mexiquense de Estudios Psicopedagógicos de Zumpango.

Basto, R. (2014). La superación profesional pedagógica asociada al trabajo de formación vocacional y orientación profesional como expresión de la labor del departamento docente en la Educación Preuniversitaria. Revista: IPLAC, I. Sección: Experiencia Educativa. ISSN 1993-6850.

Basto, R. (2014). La preparación profesional pedagógica de los docentes, en orientación profesional, desde la labor del departamento docente en el preuniversitario. Tesis en defendida opción al grado científico de Doctor en Ciencias Pedagógicas. Santiago de Cuba: Universidad de Ciencias Pedagógicas Frank País García.

Cebreiro, B. y Fernández, M. (2004). Estudio de casos. En Salvador, F.; Rodríguez, J. L. y Bolívar, A. (Dirs): Diccionario enciclopédico de didáctica. Málaga: Aljibe.

González, F. (1990). Motivación moral en adolescentes y jóvenes. 2. ed. La Habana: Científico Técnica.

Jardinot, L. (2003a). Currículum para la formación integral y diferenciada del bachiller cubano. Informe de Investigación del Proyecto Modelo de Preuniversitario. Santiago de Cuba: ISP Frank País García.

Jardinot, L. (2003). Hacia un preuniversitario de nuevo tipo. Una experiencia de investigación curricular participativa y multidisciplinaria. La Habana: Evento Internacional Pedagogía 2003.

Mesa, N. y Salvador, R. (s/f.). Precisiones sobre el contenido y las formas del trabajo metodológico en el preuniversitario. En www.monografias.com. Consultado el 20 de marzo de 2012.

Mesa, N. y otros. (2004). Hacia la integración del trabajo metodológico, la superación y la investigación en el preuniversitario. Santa Clara: Universidad de Ciencias Pedagógicas Félix Varela.

(2007). La superación de los profesores del preuniversitario en las condiciones de las transformaciones en la educación. Santa Clara. Universidad de Ciencias Pedagógicas Félix Varela.

Sierra, R. (2004). Modelación y estrategia: algunas consideraciones desde una perspectiva pedagógica. En: Compendio de Pedagogía. Ed: Pueblo y Educación. Ciudad de La Habana. Cuba. 